\title{
Exploring Motives for Using Facebook: A Latent Profile Analysis
}

\author{
Irina CRISTESCU, Alexandru BALOG* \\ National Institute for Research and Development in Informatics - ICI Bucharest, \\ 8-10 Averescu Ave., Bucharest, 011455 Romania \\ e-mail: irina.cristescu@ici.ro, alexandru.bolog@ici.ro (*Correspondingauthor)
}

\begin{abstract}
People use Facebook from a variety of motives and literature is dominated by descriptive statistics and variableoriented approaches (e.g., regression). The aim of this paper is to extend previous research by adopting a person-oriented approach (i.e., LPA - latent profile analysis) and examine whether profiles of motivation can be identified. Two empirical studies were performed engaging Romanian university students (557 and 550, respectively). The LPA and mclust package version 5.3 were used to identify groups of students who share similar response patterns to multiple facets of motivation when using Facebook. The results of LPA revealed the presence of three profiles, reflecting different levels of motivations for using Facebook: low, moderate, and high. These profiles differed significantly from each other with respect to maintaining social relationships, extending social relationships, and information \& collaboration. The results illustrate that maintaining social relations is the most important factor to create the profiles. Also, students in the high motivational profile reported more time spent on Facebook than those in the other two profiles.
\end{abstract}

Keywords: Facebook, Motivations, Motivational profile, Latent profile analysis, Mclust.

\section{Introduction}

Online social networks, including Facebook, are widely used by university students. In order to benefit from the educational potential of the Facebook it is important to understand the reasons or motives why students are using it.

Almost all research regarding Facebook uses employed variable-oriented approaches (e.g., regression, factor analysis) in examining the motives for using Facebook and their effects on various behavioral variables. These approaches are correct, assuming that the samples are homogeneous and that findings can apply to all individuals. However, motivations may change over time and it is common for individuals to report a combination of multiple motives for using Facebook. Heterogeneity is often investigated by looking at the relationships among individuals (i.e., person-oriented approach [6]) instead of variables. In this respect, person-oriented approach (e.g., model-based clustering, latent profile analysis) has the advantage of providing more intuitive ways of understanding motivations in terms of groups or individuals' profiles.

In this research, a person-oriented approach (i.e., latent profile analysis) was used to identify groups of students who share similar response patterns to multiple motivations for using Facebook. The present research is the first to empirically assess the motivations for using Facebook on the basis of the latent profile analysis technique. Thus, the current contribution to the literature consists in using advanced statistical modeling methods for identifying the profiles of motives presented in two samples of Romanian students.

The paper is structured as follows. Section 2 presents the related work with a focus on the motives of Facebook use and user typologies. Section 3 describes the present research and the analytical procedures used. The results and analyses are presented in Sections 4 and 5. The paper ends with the discussion and conclusions in Sections 6 and 7, respectively.

\section{Related Work}

Relevant studies show that people, including university students, use Facebook from a variety of motives. For example: friendship, relationship formation $[11,17,25]$; information seeking / searching $[4,11,12,18]$; relationship maintenance $[12,15,22,25]$; keeping in touch with friends [18]; entertainment $[4,11,17]$ etc. The authors have used different terms to express similar meanings. As pointed out by Yang \& Brown (2013), "studies have not converged on a comprehensive list of the major motives underlying college students' use of Facebook". Previous literature regarding the motives for using Facebook is dominated by descriptive statistics and variable-oriented approaches such as regression, factor analysis, and structural equation modeling which focus on describing the relationships among variables (e.g., motives for using social networking sites, antecedents, and consequences). 
Also, literature reports a number of typologies of reasons and ways that people use social networks (e.g., $[1,2,3,21])$. Attention has shifted from variable-oriented approaches towards personoriented approaches which include methods such as cluster analysis, latent class analysis, latent profile analysis. The focus is on the relationships among individuals, and the goal is to classify individuals into distinct groups or classes based on individual response patterns so that individuals within a group are more similar than individuals between groups. For example, Shao et al. (2015) developed a motivation-based segmentation typology and identified four clusters of Facebook users in Australia: devotee, agnostic, socialiser, and finder. Alarcon-del-Amo et al. (2015) used a latent segmentation approach to obtain a typology based on the frequency with which Spanish and Dutch users perform different activities and experience in social networking. Recently, by using Two Step Cluster procedure, Bulut \& Dogan (2017) identified four users groups in Turkey: advanced users, business-oriented users, communication seekers, and dawdlers. Scott et al. (2017) conducted latent profile analyses in a sample of US adults to explore their social media use in terms of frequency (how much time they spend online) and engagement (how youth spend their time online). More recently, in Romania, Cristescu \& Balog (2018) used the Two Step Cluster Analysis and identified three motivational different groups: high, moderate, and low. Lo Coco et al. (2018) determined homogeneous groups of Facebook users based on variables regarding personal experience on Facebook, by using a latent class analysis. In short, empirical literature in the area of online social networks is limited to a few studies that have applied personoriented approaches.

\section{The Present Research}

In the present research we have used a personoriented approach (i.e., LPA, latent profile analysis) to identify and compare subgroups of students that differ with regard to motives for using Facebook. LPA is a special case of the latent variable mixture modeling [6], known in the literature also as latent class cluster analysis and model-based clustering. LPA allows for the identification of unique subgroups based on the profiles indicated by continuous variables $[6,24]$. In the last decade, LPA has been frequently used in domains such as education, health, information systems, management, marketing and psychology.

In this research, LPA results in a typology in which university students are classified into quantitatively and qualitatively distinct profiles based on their specific configuration on a set of variables (i.e., motives for using Facebook). We have used mclust package, a R package for modelbased clustering and classification [8].

No study has used before latent profile analysis with mclust to examine and to identify empiricallyderived homogeneous groups of individuals based on their motives for using Facebook. A LPA was chosen because it has several advantages over other methods, namely: it is a model-based technique, uses rigorous statistical criteria to determine the number of profiles, can accommodate variables with different scale types $[14,16,23]$.

\subsection{Sample and Measures}

The data were obtained as part of a larger research project on Facebook usage applied to Romanian students. The questionnaire has been administrated to university students between March - June 2015. Participation was voluntary and anonymous, and no incentives were offered to take part in the study. The questionnaire was administrated as a paper-pencil questionnaire.

The questionnaire included background information such as gender, age, faculty, year of study, number of friends, frequency and duration of Facebook use. Several motives for using Facebook were measured with items on a 7-points scale $(1=$ "strongly disagree" to $7=$ "strongly agree"). The scale and the model tested and validated by Iordache \& Pribeanu (2016) were used to measure three major factors related to the reasons or motives for using Facebook: maintaining social relations (MSR), extending social relations (ESR), and information \& collaboration (IC). The scale includes 9 items that assess motives for maintaining social relations (e.g., "I use Facebook to keep in touch with former high school colleagues"; three items), extending social relations (e.g., "I use Facebook to get in touch with new people"; two items), and information \& collaboration (e.g., "I use Facebook to get access to shared resources"; four items). Additionally, in order to validate our solution, we used the time spent on Facebook ("On average, how many minutes per day do 
you actually use Facebook?"). After eliminating incomplete questionnaires, there were 1126 useful questionnaires left.

\subsection{Analytical Procedures}

Prior to conducting the analyses, a SPSS 23.0 software was used to divide the original sample (1126) randomly into two parts (568 and 558, respectively), each approximating a 50\% proportion. For the considered variables, both univariate and multivariate outliers were searched in each data sample. A total of 19 cases were removed due to multivariate outliers, 11 from the first sample and 8 from the second sample. Thus, the first sample consisted of 557 valid cases and the second sample included 550 valid cases. Also, data normality was investigated in terms of skewness and kurtosis [9]. All values were within the recommended levels, supporting the moderate departure from normality.

The present research was guided by recent methodological development in the literature $[14,23]$. Thus, the first sample $(\mathrm{N}=557)$ was used to identify students' motivational profiles on the basis of their scores on the different motives for using Facebook (Study 1). The second sample $(\mathrm{N}=550)$ was used in Study 2 to replicate the motivational configurations and cross-validate results found in Study 1. As noted by Lubke \& Luningham (2017), "validation in a new sample is usually the strongest type of validation". Descriptive statistics of the variables are showed in Table 1.

Table 1. Descriptive statistics

\begin{tabular}{|c|c|c|}
\hline & $\begin{array}{c}\text { Study } 1 \\
\left(\mathrm{n}_{1}=557\right)\end{array}$ & $\begin{array}{c}\text { Study } 2 \\
\left(\mathrm{n}_{2}=550\right)\end{array}$ \\
\hline & Mean (SD) & Mean (SD) \\
\hline MSR & $5.14(1.29)$ & $5.10(1.55)$ \\
\hline ESR & $3.87(1.58)$ & $3.85(1.23)$ \\
\hline $\mathrm{IC}$ & $4.59(1.22)$ & $4.66(1.51)$ \\
\hline Time spent & $88.34(87.18)$ & $84.30(79.64)$ \\
\hline Age (years) & $21.52(2.89)$ & $21.42(2.78)$ \\
\hline \multicolumn{3}{|l|}{ Gender } \\
\hline Male & $\mathrm{n}=280(50.3 \%)$ & $\mathrm{n}=279(50.7 \%)$ \\
\hline Female & $\mathrm{n}=277(49.7 \%)$ & $\mathrm{n}=271(49.3 \%)$ \\
\hline
\end{tabular}

In the first stage, a confirmatory factor analysis (CFA) was used with AMOS 23, in each sample, to test the three factors structure and to validate the scales. The score for the individual scales were computed by taking the mean of items that make up the scale. Multiple indices were used to evaluate the model fit [8]: chi-square $\left(\chi^{2}\right)$, chisquare / degrees of freedom $(<5.0)$, the TuckerLewis index (TLI $>$.90), the comparative fit index (CFI $>.90)$, the standardized root mean residual (SRMR >.06), the root mean square error of approximation (RMSEA < .08).

The fit indices revealed a good fit of the model to the data. For the first sample, $\chi^{2}=67.90$, df $=24, \mathrm{p}<.001, \chi^{2} / \mathrm{df}=2.83$, TLI $=.95, \mathrm{CFI}=.96$, $\mathrm{RMSEA}=.06, \mathrm{CI}=[.042 ; .074]$, SRMR $=.04$; for the second sample $\chi^{2}=98.84, \mathrm{df}=24, \mathrm{p}<.001, \chi^{2} /$ $\mathrm{df}=4.12, \mathrm{TLI}=.91, \mathrm{CFI}=.94, \mathrm{RMSEA}=.07,90 \%$ $\mathrm{CI}=[.060 ; .091], \mathrm{SRMR}=.05$. The three scales had acceptable internal consistency (Cronbach's $\alpha>$.60). In each sample, all factor loadings were found to be significant and greater than 0.50, and the average variances extracted (AVE) for each factor exceeded 0.50. Also, all squared correlations between paired factors did not exceed the AVE for each of the paired factors. Therefore, the three-factor structure indicated convergent and divergent validity in the two samples and it was used in the following stage. Results of these tests are available upon request.

In the second stage, a latent profile analysis (LPA) and mclust package version 5.3 in $\mathrm{R}$ 3.4.1 environment [8] were used to investigate the optimal number of latent profiles (groups) that describe the students' motives for using Facebook. In mclust, the number of components and the parameters are selected using the Bayesian Information Criterion (BIC). The optimal solution considered fourteen possible models with different geometric characteristics (volume, shape and distribution), according to [20]. Based on the recommendations in the literature (e.g., Pastor et al., 2007; Stanley et al., 2017; Scrucca et al., 2016) several criteria were used to select the optimal solution: (a) the highest BIC value; (b) a non-significant LRTS $p$ value $(>.05)$; (c) profile with high estimated posterior probabilities; (d) no profile contains less than $5 \%$ of the students; (e) each profile has theoretical meaningfulness. The accuracy of the optimal solution was assessed by examining the entropy statistics, computed by the authors according to the formula in [16], and posterior probabilities. The profiles' names were defined according to the obtained means. 
Next, by using GLM procedure in SPSS 23, a oneway multivariate analysis of variance (MANOVA) was conducted to test whether the motives for using Facebook differed significantly across the three profiles. Finally, in order to provide external validity evidence for the optimal solution, an oneway ANOVA was conducted with time spent on Facebook as the dependent variable and with profile membership as the independent variable.

\section{Study 1 - Identified Profiles}

We used LPA and mclust package with the three factors as variables (MSR, ESR, and IC). The best fit was obtained for an EVI (diagonal, equal volume, varying shape) model with three components. A summary showing the top-three models is presented in Table 2 .

Table 2. Best BIC values $\left(\mathrm{n}_{1}=557\right)$

\begin{tabular}{|l|r|r|r|}
\hline & EVI,3 & EVE,3 & EEI,4 \\
\hline BIC & -5542.213 & -5551.533 & -5554.312 \\
\hline BIC diff & 0.000 & -9.319 & -12.099 \\
\hline
\end{tabular}

The three-profile solution had the highest BIC value. Moreover, the LRTS $p$-value was significant $(p=.080)$ and indicated that the four-profile model was to be rejected in favour of the three-profile model. A plot of BIC for all models considered is illustrated in Figure 1.

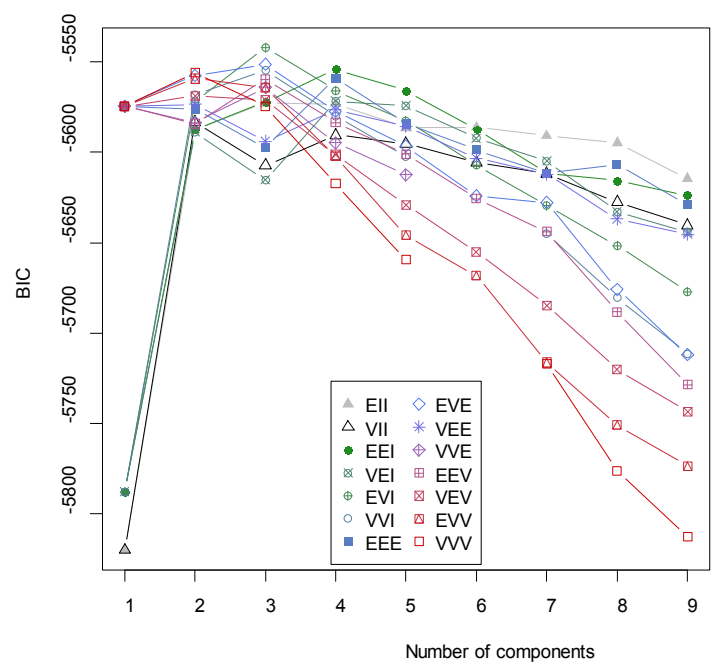

Figure 1. BIC plot for fitted models (study 1)

A contour plot of estimated densities is illustrated in Figure 2.

The profile size and average posterior probabilities support the solution with three profiles. Results in Table 3 indicate that there are sufficient numbers of cases in each of the three profiles ranging from
114 to 257 . The probabilities that students belong to their assigned profiles were high (0.81-0.88), whereas probabilities that students belong to other profile were low $(0.02-0.15)$.

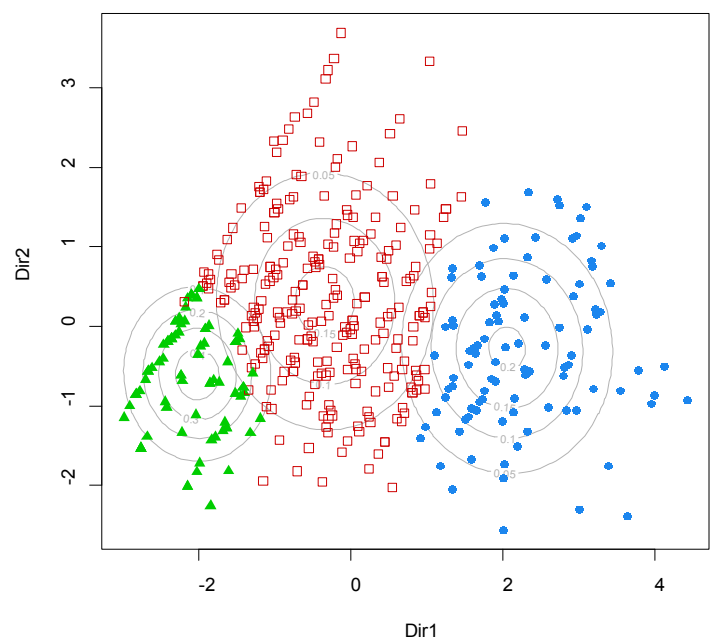

Figure 2. Plot of estimated densities (study 1)

However, there is some slight overlap between profiles 1 and 2 . The value of the entropy statistics was moderate in value (0.69).

Table 3. Posterior probabilities $\left(\mathrm{n}_{1}=557\right)$

\begin{tabular}{|l|c|c|c|c|}
\hline Profile & $n$ & 1 & 2 & 3 \\
\hline 1 & 114 & $\mathbf{0 . 8 1}$ & 0.15 & 0.04 \\
\hline 2 & 186 & 0.06 & $\mathbf{0 . 8 6}$ & 0.08 \\
\hline 3 & 257 & 0.02 & 0.10 & $\mathbf{0 . 8 8}$ \\
\hline
\end{tabular}

The results previously mentioned were used to name the profiles (see Table 4). The profiles' names were defined according to the obtained means. The first latent profile was named "low" profile because $19.2 \%$ (114) of the students reported the lowest levels on all motives for using Facebook. The second profile named "moderate" profile, comprised $36.7 \%$ (186) of the students with moderate levels in MSR and IC, but slightly lower level of ESR. Finally, the third profile named "high" profile, contained $44.1 \%$ (257) of the students who reported high levels on all motives.

Table 4. Profiles of motivations $\left(n_{1}=557\right)$

\begin{tabular}{|l|c|c|c|}
\hline & $\begin{array}{c}\text { Low } \\
19.2 \% \\
(\mathrm{n}=114)\end{array}$ & $\begin{array}{c}\text { Moderate } \\
36.7 \% \\
(\mathrm{n}=186)\end{array}$ & $\begin{array}{c}\text { High } \\
(\mathrm{n}=1 \%\end{array}$ \\
\hline MSR & 4.15 & 4.43 & 6.16 \\
\hline ESR & 1.82 & 3.81 & 4.80 \\
\hline IC & 3.64 & 4.31 & 5.24 \\
\hline
\end{tabular}


To ensure that the profiles are clearly differentiated, a one-way multivariate analysis of variance (MANOVA) was conducted using profile membership as the independent variable and the motivational variables (MSR, ESR, IC) as the dependent variables. We found significant differences among the three profiles with respect to all of the motivational variables (Pillai's Trace $\left.=.925, \mathrm{p}<.001 ; \eta^{2}=.462\right)$. The effect size is large and represents $46.2 \%$ of the variance in motives scores explained by the profile membership. Among motives, MSR had the greatest effect on all profiles. A one-way ANOVA was conducted on each dependent variable as a follow-up to the MANOVA. The ANOVAs were all significant: $\mathrm{F}(2,554)=353.98, \mathrm{p}<.001, \eta^{2}=0.561 ; \mathrm{F}(2,554)$ $=318.42, \mathrm{p}<.001, \eta^{2}=0.535 ; \mathrm{F}(2,554)=108.53$, $\mathrm{p}<.001 \eta^{2}=0.282$. The final profile solution accounted for $56.1 \%, 53.5 \%$, and $28.2 \%$ of the variance in each motivational variable (MSR, ESR, and IC, respectively). The results of Tamhane's $\mathrm{T}$ post hoc tests revealed that all three profiles differed significantly from each other on each of the motivations, except for the "Low" profile which did not differ from "Moderate" profile on MSR variable. However, overall, these differences support the distinction among the three profiles.

The association between profile membership and time spent on Facebook (minutes per day) were investigated. A one-way ANOVA showed that the profiles differed in the amount of time spent on Facebook for the three profiles: $\mathrm{F}(2,554)=6.381$, $\mathrm{p}<.05, \eta^{2}=.023$. Students belonging to "High" profile spend more time $(\mathrm{M}=98.51, \mathrm{SE}=5.39)$ than other students who belong to "Moderate" profile $(\mathrm{M}=89.31, \mathrm{SE}=6.33)$ and "Low" profile $(\mathrm{M}=63.84, \mathrm{SE}=8.09)$. However, "Moderate" and "High" profile did not differ significantly.

\section{Study 2 - Cross-validation}

As in Study 1, a LPA and mclust package with the three factors as variables were used. The best fit was obtained for a VII (spherical, varying volume) model with three components. A summary showing the top-three models is presented in Table 5.

Table 5. Best BIC values $\left(\mathrm{n}_{2}=550\right)$

\begin{tabular}{|l|r|r|r|}
\hline & VII,3 & VEI,3 & VVI,3 \\
\hline BIC & -5535.45 & -5536.62 & -5538.92 \\
\hline BIC diff & 0.000 & -1.17 & -3.47 \\
\hline
\end{tabular}

The three-profile solution had the highest BIC. LRTS $p$-value was significant $(p=.065)$ and indicated that the four-profile model was to be rejected in favour of the three-profile model.

The plots of BIC and of estimated densities are shown in Figure 3 and Figure 4, respectively.

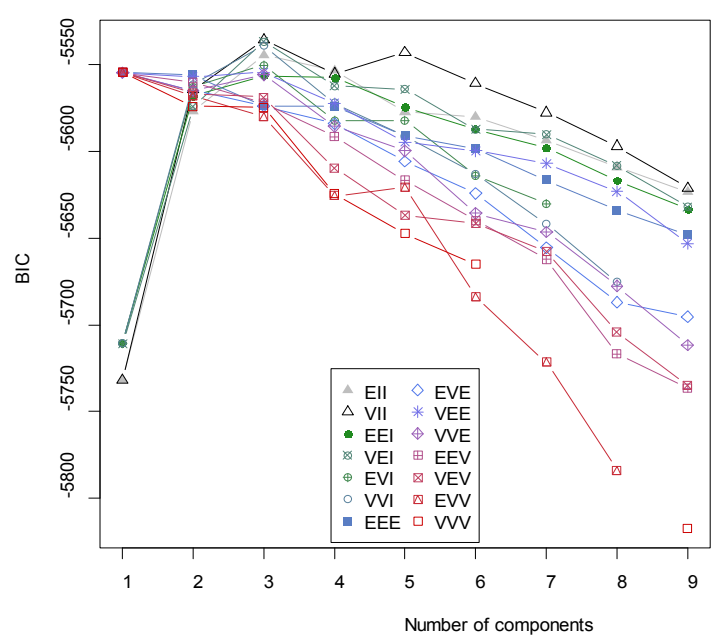

Figure 3. BIC plot for fitted models (study 2)

The value of the entropy statistics (0.72) indicated a good classification accuracy.

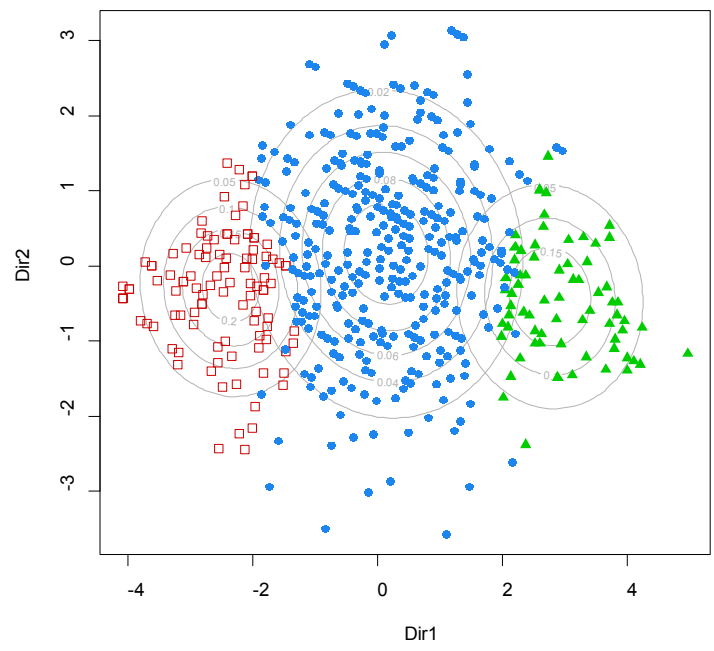

Figure 4. Plot of estimated densities (study 2)

Also, the results from Table 6 indicated that there are sufficient numbers of cases in each of the three profiles ranging from 71 to 375 . Students in all groups have a high probability $(\geq 0.77)$ of being correctly classified into a group and a low probability $(\leq 0.23)$ of being incorrectly classified. There is some overlap between profiles 1 and 2, and between profiles 2 and 3 .

Table 6. Posterior probabilities $\left(\mathrm{n}_{2}=550\right)$

\begin{tabular}{|l|c|c|c|c|}
\hline Profile & $n$ & 1 & 2 & 3 \\
\hline 1 & 71 & $\mathbf{0 . 7 7}$ & 0.23 & 0.00 \\
\hline 2 & 375 & 0.04 & $\mathbf{0 . 9 0}$ & 0.06 \\
\hline 3 & 104 & 0.00 & 0.19 & $\mathbf{0 . 8 1}$ \\
\hline
\end{tabular}


The three profiles (see Table 7) were very similar to those identified in Study 1, with the exception of the second profile that contained moderate to high level of maintaining social relations (MSR). The profiles names were defined according to the means obtained. The first profile was named "low" profile because $12.9 \%$ (71) of the students reported the lowest levels on all motives for using Facebook. The second profile named "moderate" profile, comprised of $67.9 \%$ (375) of the students with moderate to high levels in MSR, but moderate levels of ESR and IC. Finally, the third profile named "high" profile contained 19.2\% (104) of the students who reported high levels on all motives.

Table 7. Profiles of motivations $\left(\mathrm{n}_{2}=550\right)$

\begin{tabular}{|l|c|c|c|}
\hline & $\begin{array}{c}\text { Low } \\
12.9 \% \\
(\mathrm{n}=71)\end{array}$ & $\begin{array}{c}\text { Moderate } \\
67.9 \% \\
(\mathrm{n}=375)\end{array}$ & $\begin{array}{c}\text { High } \\
19.2 \% \\
(\mathrm{n}=104)\end{array}$ \\
\hline MSR & 3.34 & 5.13 & 6.14 \\
\hline ESR & 2.09 & 3.63 & 5.78 \\
\hline IC & 3.40 & 4.63 & 5.49 \\
\hline
\end{tabular}

The results of MANOVA indicated that there are significant differences between the three profiles on the three variables used (Pillai's Trace $=.785$, $p<.001, \eta^{2}=0.393$ ). The effect size is large and represents $39.3 \%$ of the variance in motives scores explained by the profile membership. A one-way ANOVA was conducted on each dependent variable as a follow-up to the MANOVA. The ANOVAs were all significant: $F(2,547)=190.91, p<.001$, $\eta^{2}=0.411 ; F(2,547)=335.23, p<.001 ; \eta^{2}=0.551$; $F(2,547)=104.39, p<.001, \eta^{2}=0.276$. The final profile solution accounted for $41.1 \%, 55.1 \%$, and $27.6 \%$ of the variance in each motivational variable (MSR, ESR, and IC, respectively). The results of Tamhane's $T$ post hoc tests revealed that all three profiles differed significantly from each other on each of the motivations. These differences provide good support for the distinctiveness of the motivational profiles.

A separate one-way ANOVA showed that the profiles differed in the amount of time spent on Facebook for the three profiles: $\mathrm{F}(2,547)=4.61$, $\mathrm{p}<.05, \eta^{2}=.017$. Specifically, students belonging to "High" profile spend more time on Facebook $(\mathrm{M}=90.21, \mathrm{SE}=7.76)$ than other students belonging to "Moderate" $(\mathrm{M}=87.68, \mathrm{SE}=4.09)$ and "Low" profile ( $\mathrm{M}=57.82, \mathrm{SE}=9.39)$. However, "Moderate" and "High" profile did not differ significantly.

\section{Discussion}

The present study aimed to reveal motivational profiles based on motives of university students to use Facebook and to assess the differences between the derived profiles. In order to empirically validate our profiles, we performed a split-half cross-validation using two random subsamples generated from the larger data set. The profiles identified in the first sample were crossvalidated in the second sample. The LPA showed a three-profiles solution in the two samples.

Overall, in each sample, LPA revealed three quantitatively and qualitatively distinct profiles of motives for using Facebook. Based on the variables from which they were derived, the three motivational profiles were named as follows: "Low", "Moderate", and "High". The university students in the three profiles differed significantly in their motivations.

The first profile was the least motivated group of Facebook students, reporting the lowest scores on all motives for using Facebook. The "Low" profile was the smallest profile in the two samples (19.2\% and $12.9 \%$, respectively). In each sample, the scores for this group were much lower than the mean for the whole sample. All motives are considered to be less important and have a low value for members belonging to this profile. ESR is the least important in the two samples. Low scores show that for members of this group the factors are not relevant motivations in using Facebook. Probably, members of this group use Facebook for other purposes than those defined by the questionnaire used (for example, for time passage, entertainment, pleasure).

The second profile was the largest in sample 2 , and second-largest in sample 1 . This profile was the only profile whose scores were around the midpoint of 4 on all three motivations and around the average for each whole sample. As in "Low" profile, ESR is the least important in the two samples. From the comparative analysis of the average scores obtained, it can be roughly deduced that the members of this profile use Facebook mainly for maintaining social relations, but not on as priority basis.

The third profile was the most motivated group, reporting the highest scores on all motives for using Facebook. Student members belonging to this profile have a well-defined purpose for using Facebook. Members of this group have strong 
beliefs about the purpose of using Facebook. In addition to using Facebook to maintain social relationships, they use Facebook for informationcollaboration and for extending social relations. Apparently, the main reason for using Facebook is to maintain social relationships.

In Study 1, the hierarchical ordering of the three motivations does not differ across profiles. This suggests that in this sample there are only quantitative differences between students who belong to each identified profile. However, in Study 2 the hierarchical ordering of the three motivations differs across profiles. Specifically, in "Low" profile the order is IC, MSR, and ESR, in "Moderate" profile the order is MSR, IC, and ESR, and in "High" profile the order is MSR, ESR, and IC. This demonstrates that in each profile (group) the students focused on different motivations. Students have a distinct pattern of motivations on which they are low, moderate, or high. In other words, in this sample heterogeneity is high and there are quantitative and qualitative differences between students belonging to each identified profile.

The results indicate that maintaining social relations is the most important factor to create the profiles. One of the explanations for which MSR has the greatest effect on all groups may be due to the feeling of belonging that is fulfilled when students use Facebook to communicate with strong ties (friends and old friends or colleagues). Social ties help students become socially integrated and require a constant effort for keeping the relationship alive. Thus, frequent communication not only increases liking, but also provides opportunities for self-disclosure and social support, which deepen the relationships [5].

The results from both studies show an association between the profiles and the time spent on Facebook. Students in the high motivational profile reported more time spent on Facebook than those in the other two profiles. The results confirm and are in line with previous research findings $[4,19]$. Together, our findings suggests that latent person-oriented approach, such as LPA, is a useful tool for studying student Facebook motivations.

Although this research contributes to the literature, several potential limitations create a future research avenue. First, the sample is limited to a convenience, cross-sectional sample and the study took place in Romania, making any generalization of the results difficult. Therefore, cross-cultural studies should also be conducted in the future using different groups from different countries and cultures. Second, other motives for using Facebook identified in the literature (e.g., entertainment, passing time) were not included in this study. There is a need for further research to include more variables related to motivation for Facebook usage. Third, motives and latent profiles were analyzed only in relation to time spent on Facebook. Future research should also consider other outcomes or consequences of motivational profiles (e.g., Facebook addiction, academic performance, well-being, social capital).

\section{Conclusion}

The current study adopted a person-oriented approach (i.e., latent profile analysis) to identify groups of students who shared similar response patterns to multiple facets of motivation for using Facebook. As needs, purposes, and motivations may change over time, future research should investigate other configurations of motives and identify possible changes in the motivational profiles (e.g., by using latent transition analysis [6]), extending thus the scope of the present study.

\section{Acknowledgements}

This research was supported by a national grant financed by The National Authority for Scientific Research (ANCS), Romania, under COGNOTIC 1609 0101/2016-2017.

\section{REFERENCES}

1. Alarcón-del-Amo, M-C., Gómez-Borja, M-Á. \& Lorenzo-Romero, C. (2015). Are the users of social networking sites homogeneous? A cross-cultural study, Frontiers in Psychology, 6, 1127.

2. Antoniadis, I., Koukoulis, I. \& Serdaris, P. (2015). Social network sites' usage among Greek students in Western Macedonia, Int. J. Strategic Innovative Marketing, 3, 52-66.

3. Brandtzaeg, P. B. \& Heim, J. (2011). A typology of social networking sites users, International Journal of Web Based Communities, 7(1), 28-51.

4. Bulut, Z. A. \& Doğan, O. (2017). The ABCD typology: Profile and motivations of Turkish social network sites users, Comp. in Hum. Behav., 67(2), 73-83. 
5. Burke, M. \& Kraut, R. E. (2016). The relationship between Facebook use and wellbeing depends on communication type and tie strength, Journal of Computer-Mediated Comm., 21(4), 265-281.

6. Collins, L. M. \& Lanza, S. T. (2010). Latent class and latent transition analysis: With applications in the social, behavioral, and health sciences. NJ, John Wiley \& Sons.

7. Cristescu, I. \& Balog, A. (2018). Motives for using Facebook among university students: A Two-Step Cluster Analysis. In Proc. of the $17^{\text {th }}$ Intl. Conf. On Informatics in Economy (IE 2018) (pp. 257-262).

8. Fraley, C., Raftery, A. E., Scrucca, L., Murphy, T. B. \& Fop, M. (2017). mclust: Gaussian mixture modelling for modelbased clustering, classification, and density estimation. R package version 5.3.

9. Hair, J. F., Black, W. C., Babin, B. J. \& Anderson, R. E. (2014). Multivariate Data Analysis. 7th Pearson Education Limited.

10. Iordache, D. D. \& Pribeanu, C. (2016). Exploring the motives of using Facebook - a multidimensional approach, Rev. Romana de Interact. Om-Calculator, 9(1), 19-34.

11. Kim, Y., Sohn, D. \& Choi, S. M. (2011). Cultural difference in motivations for using social network sites: A comparative study of American and Korean college students, Comp. in Hum. Behav., 27(1), 365-372.

12. Ku, Y. C., Chen, R. \& Zhang, H. (2013). Why do users continue using social networking sites? An exploratory study of members in the United States and Taiwan, Information \& Management, 50(7), 571-581.

13. Lo Coco, G., Maiorana, A., Mirisola, A., Salerno, L., Boca, S. \& Profita, G. (2018). Empirically-derived subgroups of Facebook users and their association with personality characteristics: a Latent Class Analysis, Comp. in Hum. Behav., 86, 190-198.

14. Lubke, G. H. \& Luningham, J. (2017). Fitting latent variable mixture models. Behaviour Research and Therapy, 98, 91-102.

15. Park, N. \& Lee, S. (2014). College students' motivations for Facebook use and psychological outcomes, J. Broadcast Electron. Media, 58(4), 601-620.
16. Pastor, D. A., Barron, K. E., Miller, B. J. \& Davis, S. L. (2007). A latent profile analysis of college students' achievement goal orientation, Contemporary Educational Psychology, 32(1), 8-47.

17. Pornsakulvanich, V. \& Dumrongsiri, N. (2013). Internal and external influences on social networking site usage in Thailand, Comp. in Hum. Behav., 29(6), 2788-2795.

18. Raacke, J. \& Bonds-Raacke, J. (2008). MySpace and Facebook: Applying the uses and gratifications theory to exploring friend-networking sites, CyberPsychology \& Behavior, 11(2),169-174.

19. Scott, C. F., Bay-Cheng, L. Y., Prince, M. A., Nochajski, T. H. \& Collins, R. L. (2017). Time spent online: Latent profile analyses of emerging adults' social media use, Comp. in Hum. Behav., 75, 311-319.

20. Scrucca, L., Fop, M., Murphy, T. B. \& Raftery, A. E. (2016). mclust 5: Clustering, classification and density estimation using gaussian finite mixture models, The $R$ journal, 8(1), 289-317.

21. Shao, W., Ross, M. \& Grace, D. (2015). Developing a motivation-based segmentation typology of Facebook users, Marketing Intelligence \& Planning, 33(7), 1071-1086.

22. Sheldon, P. (2008). The Relationship between unwillingness-to-communicate and students' Facebook use, Journal of Media Psychology, 20(2), 67-75.

23. Stanley, L., Kellermanns, F. W. \& Zellweger, T. M. (2017). Latent profile analysis: Understanding family firm profiles, Family Business Review, 30(1), 84-102.

24. Vermunt, J. K. \& Magidson, J. (2002). Latent class cluster analysis, in Hagenaars, J. \& McCutcheon, A. (Eds.), Applied latent class analysis, 89-106. Cambridge: Cambridge University Press.

25. Yang, C. C \& Brown, B. B. (2013). Motives for using Facebook, patterns of Facebook activities, and late adolescents' social adjustment to college, Journal of Youth and Adolescence, 42(3), 403-416. 